\title{
Integração de Story Points e Use Case Points em Projetos Baseados em SCRUM e CMMI
}

\author{
Ana Sofia C. Marçal ${ }^{1,2}$, Carla Ilane Moreira Bezerra ${ }^{1,2}$, Carlo Giovano da Silva \\ Pires $^{1}$, Ciro Coelho ${ }^{1}$, Gabriela Telles de Souza ${ }^{1}$, Joice Barbosa ${ }^{1}$ \\ ${ }^{1}$ Instituto Atlântico \\ Rua Chico Lemos, 946 - Cidade dos Funcionários - Fortaleza - Ceará - Brazil \\ ${ }^{2}$ Universidade de Fortaleza (UNIFOR) \\ Av. Washington Soares, 1321 - Fortaleza - Ceará - Brazil \\ \{marcal_sofia, carlailane, cgiovano, ciro, gabi, \\ barbosa_joice\} eatlantico.com.br
}

\begin{abstract}
Developing reliable estimates is one of the most critical aspects in software development. The adoption of agile methods and small iterations bring new challenges to estimation processes and the CMMI model implementation, such as low information availability or low level of technical detail, high team involvement in the estimation process and a tight schedule to come up with estimates. The case presented in this paper describes how Instituto Atlântico has implemented Story Points estimates in a context of high maturity CMMI level (levels 4 and 5) using Use Case Points based historical data.
\end{abstract}

Resumo. Estimativas confiáveis configuram um dos aspectos mais críticos para o desenvolvimento de software. Ao mesmo tempo, a adoção de métodos ágeis e ciclos curtos trazem novos desafios para os processos de estimativas e a utilização do modelo CMMI. Entre eles podemos citar a baixa disponibilidade de informações ou detalhamento técnico do escopo, o amplo envolvimento de todo o time no processo de estimativas e o prazo curto para obtenção de estimativas. A experiência relatada mostra como o Instituto Atlântico integrou estimativas em Story Points ao seu contexto de implantação de práticas de alta maturidade do CMMI (Níveis 4 e 5) e a uma base histórica baseada em Use Case Points.

\section{Introdução}

A melhoria dos processos de desenvolvimento de software é fundamental para as organizações que implementam programas de qualidade. Dentre os programas de qualidade mais utilizados nas organizações estão o modelo CMMI [Chrissis 2006] e a metodologia Six Sigma [Tayntor 2003].

O surgimento de vários métodos ágeis no final dos anos 90 contribuiu para uma tendência de desenvolvimento ágil de aplicações de forma que nos últimos anos as organizações vêm enfrentando um grande desafio para a integração entre métodos ágeis e modelos de maturidade. As organizações objetivam obter os benefícios da abordagem 
ágil garantindo ao mesmo tempo a aderência ao CMMI. Neste sentido, diversos estudos e esforços têm sido feitos na tentativa de combinar práticas ágeis e CMMI.

O Instituto Atlântico é uma instituição de pesquisa e desenvolvimento localizada em Fortaleza-Ceará e possui nível 3 de maturidade no modelo CMMI-SW e norma ISO 9001:2000. Visando a melhoria contínua dos seus processos, o Atlântico encontra-se em processo de implantação dos níveis 4 e 5 de maturidade do modelo CMMI com o apoio da metodologia Six Sigma. Nesse contexto, foi iniciado um projeto DMADV Processos Ágeis, tendo como principal objetivo melhorar a produtividade dos projetos por meio da adoção de implantação de práticas e estimativas ágeis. Durante a implantação deste projeto foi identificada a necessidade de conversão de estimativas ágeis a partir de Story Points (SP) em Use Case Points (UCP) de forma que os projetos usem a base quantitativa já estabelecida na organização em UCPs sem comprometer a agilidade necessária proporcionada pela estimativa em Story Points.

Esse trabalho relata a experiência do Atlântico na integração de estimativas ágeis ao contexto de implantação de práticas de alta maturidade do CMMI (Níveis 4 e 5) e está organizado em cinco seções: a seção 2 apresenta uma visão geral de Scrum e Story Points; a seção 3 descreve como está sendo realizada a inserção de processos ágeis no modelo alta maturidade do Atlântico; a seção 4 detalha o estudo de caso realizado para a integração de Use Case Points com Story Points; e a última seção apresenta os benefícios comprovados na utilização de estimativas integradas.

\section{Visão Geral de Scrum e Story Points}

Alinhado aos princípios ágeis, o Scrum foi criado em 1996 por Ken Schwaber e Jeff Sutherland, como um método que aceita que o desenvolvimento de software é imprevisível e formaliza a abstração, sendo aplicável a ambientes voláteis.

O Scrum se destaca dos demais métodos ágeis pela ênfase dada ao gerenciamento do projeto. Há atividades de monitoramento e feedback, em geral através de reuniões rápidas e diárias com toda a equipe, visando à identificação e correção de deficiências e/ou impedimentos no desenvolvimento [Schwaber 2004]. O método baseia-se ainda em princípios como: equipes pequenas de, no máximo, 7 pessoas; requisitos pouco estáveis ou desconhecidos; iterações curtas. Ele divide o desenvolvimento em períodos de no máximo 4 semanas, denominados sprints.

O fluxo de desenvolvimento do Scrum é mostrado na Figura 1. Segundo Schwaber [Schwaber 2004], cada sprint se inicia com uma reunião de planejamento (Sprint Planning Meeting), na qual o Product Owner e o Time decidem em conjunto o que deverá ser implementado (Selected Product Backlog). A reunião é dividida em duas partes. Na Sprint Planning 1, o Product Owner apresenta os requisitos de maior valor e prioriza aqueles que devem ser implementados. O Time então define colaborativamente o que poderá entrar no desenvolvimento da próxima sprint, considerando sua capacidade de produção.

De acordo com Marçal e seus colegas [Marcal 2007], a estimativa de complexidade por Story Points deve ser introduzida ao processo de estimativa e priorização dos itens do Product Backlog na Sprint Planning 1. A idéia por trás do método de estimativas por Story Points [Cohn 2006] é que o cliente e o time sejam parte 
fundamental das estimativas, na qual o primeiro considera o valor que cada item do Product Backlog agrega para o seu negócio, e o segundo dimensiona a complexidade de implementação de cada item baseado no contexto atual do projeto.

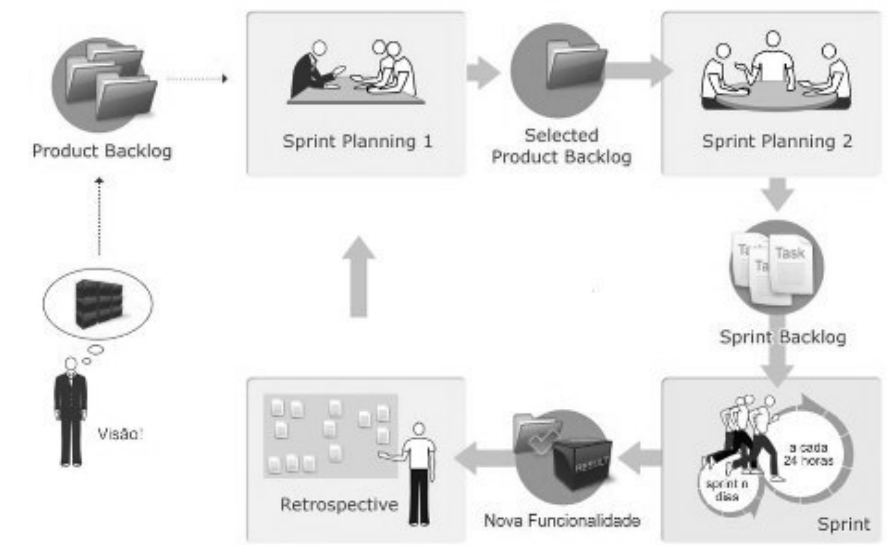

Figura 1. Visão geral do processo do Scrum [Gloger 2008].

Uma vez que todo o Product Backlog esteja valorado de acordo com o valor de negócio de cada item, a equipe do projeto estima a complexidade de cada um destes itens em Story Points. Para isto, podem utilizar a técnica Planning Poker, que segundo Cohn [Cohn 2006] é um método de atribuição de estimativas colaborativo, no qual cada integrante do time possui um conjunto de cartas identificadas com rótulos diferentes (por exemplo, com a sequiência de Fibonacci: 2, 3, 5, 8, 13, 21 e 34).

\section{Alta Maturidade e Processos Ágeis no Atlântico}

\subsection{Gestão Quantitativa de Projetos}

O Atlântico utiliza a metodologia Six Sigma para implantar os níveis 4 e 5 de maturidade do CMMI. Isto se deve ao controle estatístico que cobre a gestão quantitativa dos processos e projetos do nível 4, melhoria contínua atingida pela implementação dos projetos DMAIC (Define, Measure, Analyse, Improve and Control) e a inovação que pode ser coberta por outra metodologia do Six Sigma o DMADV (Define, Measure, Analyse, Design and Verify), atingindo assim práticas do nível 5.

No Atlântico, a gestão quantitativa de processos é realizada por meio de baselines de desempenho que determinam a situação atual da organização. As baselines também fornecem a base para identificação e medição de melhorias e inovações, ajudam a determinar metas organizacionais e de projetos e auxiliam no acompanhamento do desempenho dos projetos.

Atualmente, a organização trabalha com duas baselines principais: Produtividade e Densidade de Defeitos. Os aspectos representados nessas baselines (produtividade e densidade de defeitos) são chamados Y. Esses Ys são compostos por fatores que os influenciam denominados $\mathrm{X}$. Como exemplo, temos o percentual de problemas encontrados em revisões técnicas, no caso de densidade de defeitos, e o grau de instabilidade de requisitos, no caso de produtividade.

Vários dos Ys e Xs utilizados para o controle quantitativo da organização dependem diretamente de uma medida de tamanho. A produtividade, por exemplo, é 
uma relação entre esforço e tamanho, enquanto a densidade de defeitos é uma relação entre a quantidade de defeitos encontrados e o tamanho. O tamanho, atualmente, é medido em Use Case Points (UCP). Assim, a UCP é uma importante forma de normalizar os dados e manter a consistência das baselines de desempenho.

\subsection{Estimativas em Use Case Points}

A UCP é uma técnica de estimativa de tamanho de projeto de software orientado a objetos, criada por Karner [Karner 1993]. As estimativas com UCP são realizadas a partir da contagem do número de transações dos casos de uso. A quantidade de transações determina a complexidade de casos de uso, que, juntamente com a complexidade dos atores e os fatores técnicos e ambientais do projeto, determina o tamanho do produto.

O Atlântico utiliza uma extensão da UCP, denominada TUCP, proposta por Monteiro [Monteiro et al. 2005]. Em relação à UCP, a TUCP apresenta as seguintes melhorias:

- Um Guia para a elaboração de casos de uso, influenciando fortemente no cálculo do tamanho dos casos de uso.

- Detalhamento do conceito de transação no contexto de casos de uso, por afetar diretamente o cálculo dos pontos de casos de uso não ajustados.

- Criação de um novo nível de complexidade para casos de uso complexos (N-Complexos), para evitar possíveis subestimativas, especialmente quando o número de transações é muito grande.

- Exclusão dos fatores técnicos de ambiente (EF) do cálculo do tamanho, por se entender que o tamanho é uma grandeza física que não deveria ter seu valor alterado em função dos fatores técnicos de ambiente.

- Detalhamento do cálculo do esforço nas etapas do ciclo de vida.

- Granularização do cálculo do tamanho do projeto por cada etapa do processo de desenvolvimento e por caso de uso. na Tabela 1.

O cálculo da complexidade em TUCP, a partir das transações, é feito com base

Tabela 1. Complexidade dos casos de uso $\mathrm{x}$ transações

\begin{tabular}{|l|l|}
\hline Complexidade & Número de Transações \\
\hline Simples & Até 3 transações \\
\hline Intermediário & De 4 a 7 transações \\
\hline Complexo & De 8 a 11 transações \\
\hline N-Complexo & A partir 12 transações \\
\hline
\end{tabular}

\subsection{Projeto DMADV Processos Ágeis}

O projeto DMADV Processos Ágeis foi iniciado a partir de uma necessidade identificada no planejamento estratégico anual da organização. O principal objetivo do projeto inclui a melhoria da produtividade dos projetos por meio da adoção de um processo mais enxuto e ágil e que, ao mesmo tempo, seja aderente ao CMMI. 
$\mathrm{Na}$ fase Definir foi estabelecida, a partir da baseline de desempenho da organização, uma meta de melhoria de produtividade para o projeto. Foram também levantadas práticas ágeis com potencial para melhorar a produtividade. $\mathrm{Na}$ fase Medir, foram coletados os dados da situação da organização e dos principais fatores que influenciam a produtividade. Esses dados serviram de base para a identificação, na fase Analisar, das soluções com maior potencial de melhoria da produtividade. Após diversas análises estatísticas e pesquisas com participantes de projetos, foram priorizadas, para implantação em um primeiro momento, práticas ágeis de análise e projeto, gestão de projetos e gestão de requisitos.

$\mathrm{Na}$ fase Projetar, foi definido como as práticas selecionadas deveriam ser aplicadas nos projetos. As práticas de análise e projeto adotadas seguem os princípios da Modelagem Ágil [Ambler 2002]. Já as práticas de gestão de projetos e gestão de requisitos seguem o processo definido pelo Scrum, incluindo a estimativa pela equipe com base em Story Points. Para manter a compatibilidade dessa técnica de estimativa com a base histórica e as baselines de desempenho da organização, foi elaborado um método de conversão de Story Points para UCPs, conforme apresentado na seção 4.

Atualmente o projeto encontra-se na fase Verificar, onde as melhorias estão sendo incorporadas ao processo da organização e os resultados estão sendo medidos.

\section{Integração de Use Case Points com Story Points}

A proposta de integração de Use Case Points com Story Points está sendo aplicada em um projeto real de desenvolvimento de um sistema de Gestão de Suprimentos, parte integrante de uma solução de ERP (Enterprise Resource Planning) para um cliente da indústria têxtil situado no estado do Ceará.

O projeto iniciado em agosto de 2008 tem duração de 7 meses e conta com um time de cerca de 10 pessoas. O projeto usa práticas de gestão ágil do Scrum inseridas no contexto de alta maturidade, com um processo aderente ao nível 5 do CMMI. Todo o projeto é realizado incremental e iterativamente, com sprints de 4 semanas e entregas de funcionalidades ao final de cada sprint.

O projeto possui requisitos extremamente voláteis os quais são definidos ao longo do projeto com grande envolvimento do cliente. O Product Owner (analista de negócio do cliente) participa ativamente da construção do Product Backlog e das reuniões de Planejamento da Sprint. Durante as reuniões de Planejamento da Sprint, os requisitos priorizados são estimados em Story Points.

\subsection{Primeira Etapa do Estudo}

Visando garantir o amplo envolvimento de todo o time e o prazo curto para obtenção de estimativas, as estimativas das funcionalidades do sistema são inicialmente realizadas em Story Points durante a reunião de Sprint Planning 1. A técnica de Planning Poker é usada atrelada a sugestões iniciais construída pelo time para a estimativa em Story Points (Tabela 2) considerando as lógicas padrões de implementação dos casos de uso segundo o framework de desenvolvimento jCompany adotado no projeto. 
Tabela 2. Sugestões iniciais do time para a estimativa em SP

\begin{tabular}{|l|l|}
\hline Lógica de implementação do Caso de Uso & SP \\
\hline Pesquisa & 1 \\
\hline Tabular & 1 ou 2 \\
\hline CRUD & 3 ou 5 \\
\hline Mantém-Detalhe & 3,5 ou 8 \\
\hline Mestre-Detalhe & 8,13 e 21 \\
\hline Processamento & 34 \\
\hline
\end{tabular}

Em seguida, as estimativas em Story Points são convertidas para Use Case Points, usando-se a ferramenta organizacional para estimativas. Nesta etapa, as UCPs são calculadas derivando-se as complexidades dos casos de uso a partir das Story Points de acordo com a Tabela 3 .

Tabela 3. Complexidade dos casos de uso X Story Points

\begin{tabular}{|l|l|}
\hline SP & Complexidade do Caso de Uso \\
\hline 1,2 e 3 & Simples \\
\hline 5 e 8 & Médio \\
\hline 13 & Complexo \\
\hline 21 e 34 & N-Complexo \\
\hline
\end{tabular}

\subsection{Segunda Etapa do Estudo}

A segunda etapa do estudo consistiu em comparar e avaliar estatisticamente a conversão de Story Points em Use Case Points realizada empiricamente na primeira etapa e a partir daí construir um modelo consistente para gerar número de transações a partir de Story Points. Nesta etapa, ao invés de derivar a complexidade dos casos de uso a partir das Story Points, a complexidade foi estimada a partir da quantidade real de transações do caso de uso, seguindo o procedimento organizacional para contagem de transações conforme Tabela 1.

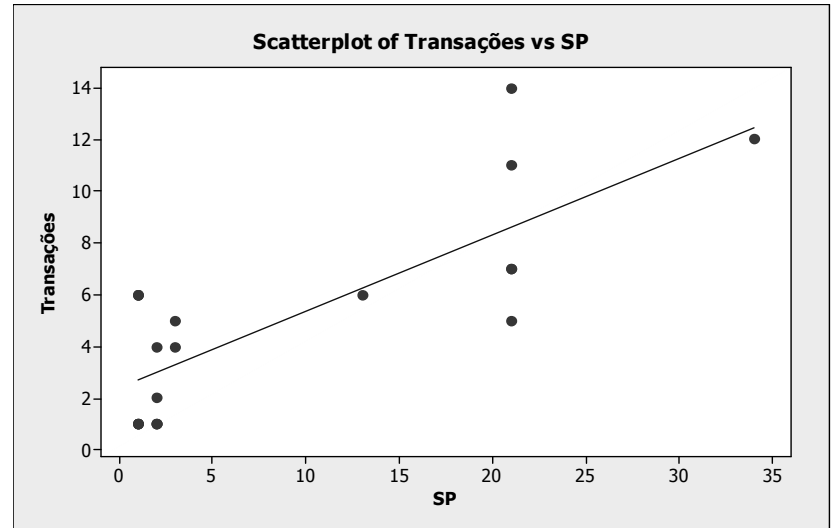

Figura 2: Análise de Regressão entre o Número de Transações e a Quantidade de Story Points.

Um conjunto de 21 casos de uso do projeto foi selecionado para compor a amostra desta segunda etapa do estudo. Para cada caso de uso da amostra foi realizada a sua contagem real de transações, derivada complexidade e calculada a sua UCP. A partir dos dados coletados (SP e contagem de transações) foi gerado o seguinte modelo de previsão usando-se a técnica de regressão linear simples: 
Transações = 2,39 + 0,296 SP (Story Points)

O gráfico de scatterplot (Figura 2) mostra graficamente a alta relação entre transações e Story Points. É importante observar que o modelo gerado é comprovado estatisticamente a um nível de significância de $1 \%$ e grau de explicação de 59,8\%.

As comparações entre as estimativas em UCPs calculadas a partir da contagem de transações e geradas a partir do modelo podem ser vistas na Tabela 4.

Tabela 4. Comparação de Estimativas (UCP Modelo x UCP Contagem)

\begin{tabular}{|c|c|c|c|c|c|}
\hline Caso de uso & SP & $\begin{array}{c}\text { Transações } \\
\text { Contagem }\end{array}$ & $\begin{array}{c}\text { Transações } \\
\text { Modelo }\end{array}$ & $\begin{array}{c}\text { UCP } \\
\text { Modelo }\end{array}$ & UCP Contagem \\
\hline Caso de uso 1 & 21 & 11 & 9 & 13,79 & 13,77 \\
\hline Caso de uso 2 & 2 & 4 & 3 & 4,60 & 9,18 \\
\hline Caso de uso 3 & 21 & 5 & 9 & 13,79 & 9,18 \\
\hline Caso de uso 4 & 21 & 7 & 9 & 13,79 & 9,18 \\
\hline Caso de uso 5 & 1 & 6 & 3 & 4,60 & 9,18 \\
\hline Caso de uso 6 & 34 & 12 & 12 & 18,39 & 18,36 \\
\hline Caso de uso 7 & 21 & 7 & 9 & 13,79 & 9,18 \\
\hline Caso de uso 8 & 21 & 14 & 9 & 13,79 & 18,36 \\
\hline Caso de uso 9 & 3 & 4 & 3 & 9,20 & 9,18 \\
\hline Caso de uso 10 & 1 & 6 & 3 & 4,60 & 9,18 \\
\hline Caso de uso 11 & 13 & 6 & 6 & 9,20 & 9,18 \\
\hline Caso de uso 12 & 3 & 5 & 3 & 9,20 & 9,18 \\
\hline Caso de uso 13 & 1 & 6 & 3 & 4,60 & 9,18 \\
\hline Caso de uso 14 & 1 & 1 & 3 & 4,60 & 4,59 \\
\hline Caso de uso 15 & 1 & 1 & 3 & 4,60 & 4,59 \\
\hline Caso de uso 16 & 2 & 1 & 3 & 4,60 & 4,59 \\
\hline Caso de uso 17 & 2 & 2 & 3 & 4,60 & 4,59 \\
\hline Caso de uso 18 & 1 & 1 & 3 & 4,60 & 4,59 \\
\hline Caso de uso 19 & 2 & 1 & 3 & 4,60 & 4,59 \\
\hline Caso de uso 20 & 2 & 1 & 3 & 4,60 & 4,59 \\
\hline Caso de uso 21 & 1 & 1 & 3 & 4,60 & 4,59 \\
\hline
\end{tabular}

\subsection{Terceira Etapa do Estudo}

Esta etapa consistiu em aplicar o modelo gerado na etapa anterior para estimar a quantidade de transações de casos de uso estimados em Story Points e a partir daí derivar a sua complexidade em Use Case Points de acordo com a Tabela 1.

A partir desta etapa as estimativas do projeto foram realizadas seguindo os passos:

1. O time realiza as estimativas de cada caso de uso em Story Points;

2. O modelo é utilizado para converter os Story Points em transações;

3. Os números de transações obtidos alimentam uma ferramenta de estimativas, já utilizada anteriormente pela organização, para o cálculo dos Use Case Points;

Os Use Case Points são utilizados para o planejamento e acompanhamento do projeto, bem como para a obtenção de dados históricos. 


\section{Conclusões, Benefícios e trabalhos futuros}

De forma geral, a utilização de Story Points e Use Case Points tem se mostrado bastante adequada, permitindo que o projeto faça uso da base quantitativa e dos processos de alta maturidade já estabelecidos na organização sem comprometer a agilidade necessária ao projeto.

A utilização do método de conversão de Story Points em Use Case Points permite a combinação dos benefícios dos dois métodos de estimativas, garantindo aumento da produtividade. Além disso, observa-se que:

- As estimativas são obtidas com maior rapidez, o que é imprescindível em um contexto de iterações curtas;

- A participação do time nas estimativas gera um maior comprometimento e permite que dificuldades técnicas sejam consideradas no momento da estimativa;

- A utilização de uma base histórica confiável torna as estimativas mais precisas;

- As estimativas obtidas seguem o padrão organizacional de UCP, garantindo a compatibilidade com a base histórica da organização, permitindo análises comparativas com outros projetos da organização e a utilização de processos de gestão de alta maturidade.

Como trabalho futuro vislumbra-se a aplicação do modelo em outros projetos da organização para o refinamento do mesmo através do aumento da amostra de casos de uso estimados em Story Points.

\section{Referências}

Ambler, S, Jeffries, R., Agile Modeling: Effective Practices for Extreme Programming and the Unified Process. Wiley, 2002.

Cohn, M. (2006) Agile Estimating and Planning, Prentice Hall, 330 p.

Chrissis, M.B., Konrad, M., Shrum, S. CMMI Second Edition Guidelines for Process Integration and Product Improvement. Version 1.2. Addison-Wesley, 2006.

Gloger, B (2007) The Zen of Scrum, http://www.glogerconsulting.de.

Karner, G. (1993) Metrics for Objectory. Diploma thesis, University of Linköping, Sweden. No. LiTH-IDA-Ex-9344:21.

Marçal, A., Freitas, B., Soares, F., Maciel, T. Belchior, A. (2007). Estendendo o SCRUM segundo as Áreas de Processo de Gerenciamento de Projetos do CMMI, CLEI 2007, San Jose, Costa Rica.

Monteiro, T. C., Pires, C. G. S., Belchior, A. D., (2005) "TUCP: Uma Extensão da Técnica UCP”, IV Simpósio Brasileiro de Qualidade de Software, Porto Alegre.

Schwaber, K. (2004) Agile Project Management With Scrum, Microsoft Press, Redmond, Washington, USA.

Tayntor, Christine B. (2003) “Six Sigma Software Development”, Flórida, Auerbach. 